
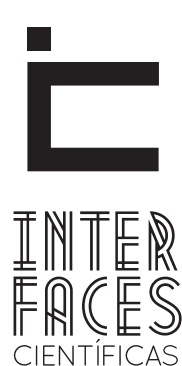

SA ÚDE E AMBIENTE

\title{
O ALEITAMENTO MATERNO NAS UNIDADES BÁSICAS DE SAÚDE DA REDE AMAMENTA BRASIL EM ARACAJU/SE
}

Liliane Santos Siqueira ${ }^{1}$

Ana Célia Goes Soares ${ }^{2}$
Renata Alves Silva ${ }^{3}$

Materno (AM), todavia a prevalência do Aleitamento Materno Exclusivo apresenta-se apenas em $40 \%$ das crianças. Também observou-se a introdução precoce de outros líquidos (água, chá e outros leites) a partir do primeiro mês de vida e de alimentos sólidos (fruta, comida salgada e outros alimentos) a partir do terceiro mês. Os dados dos questionários evidenciam que as UBS incentivam o Aleitamento Materno por meio do pré-natal, puericultura, grupos de gestante e ações educativas, assim como realizam o monitoramento desses indicadores. 0 estudo mostra que apesar das UBS promoverem ações para o AM, esta prática não acontece de forma exclusiva.

\section{PALAVRAS-CHAVE}

Aleitamento Materno. Rede Amamenta Brasil. Criança. 


\section{ABSTRACT}

Breast milk is complete food for growth and development of children up to six months, and is considered one of the main instruments for the promotion of child health. This practice is no longer given priority to families in Brazilian society since the mid-twentieth century. The study aims to describe the indicators of breastfeeding for children between 0 and 6 months of age Basic Health Units (BHU) involved in the Breastfeeding Brazil Network in the city of Aracaju / SE. We performed a cross-sectional study with data in spreadsheets Breastfeeding Network Brazil, from January to October 2012 at the BHU John and Eunice Barbosa Bezerra and from August to October 2012 at the BHU Manoel de Souza Pereira, and a structured questionnaire teams along with family health. The results show that of the 742 children studied, 643 (87\%) are breastfeeding (BF), however, the prevalence of exclusive breastfeeding provides only $40 \%$ of children. Also noted is the early introduction of other liquids (water, tea and other milk) from the first month of life and solid foods (fruits, salty foods and other foods) from the third month. The questionnaire data show that $\mathrm{BHU}$ encourage breastfeeding through prenatal care, child care, pregnant groups and educational activities, as well as track these indicators. The study shows that despite BHU promote actions for BF this practice does not occur exclusively.

\section{KEYWORDS}

Breastfeeding. Breastfeeding Network Brazil. Child.

\section{RESUMEN}

La leche materna es el alimento completo para el crecimiento y desarrollo de los niños de hasta seis meses, y es considerado uno de los principales instrumentos para la promoción de la salud infantil. Esta práctica ya no es prioridad en las familias de la sociedad brasileña desde mediados del siglo XX. El estudio tiene como objetivo describir los indicadores de lactancia materna para niños de 0-6 meses de edad de las Unidades Básicas de Salud (UBS) que participan en la red Amamanta Brasil en la ciudad de Aracaju / SE. Se realizó un estudio transversal con los datos en hojas de cálculo Red Amamanta Brasil, de enero a octubre de 2012 en la UBS John y Eunice Barbosa Bezerra y de agosto a octubre de 2012 en la UBS Manoel de Souza Pereira, así como un cuestionario estructurado junto con los equipos de salud de la familia. Los resultados demuestran que de los 742 niños estudiados, 643 (87\%) están en lactancia materna (LM), sin embargo, la prevalencia de la lactancia materna exclusiva proporciona sólo el $40 \%$ de los niños. También se incluí la introducción temprana de otros líquidos (agua, té y otras leches) a partir del primer mes de vida y alimentos sólidos (frutas, alimentos salados y otros alimentos) a partir del tercer mes. Los datos de los cuestionarios muestran que la UBS fomenta la lactancia materna a través de la atención prenatal, cuidando de niños, grupos de actividades educativas a embarazadas, así como realizar el seguimiento de estos indicadores. El estudio muestra que a pesar de UBS promover acciones para la práctica AM esto no ocurre exclusivamente.

\section{PALABRAS CLAVE}

lactancia Materna. Amamantamiento Red Brasil. Niño. 


\section{INTRODUÇ̃̃OO}

O Aleitamento Materno (AM) é um processo fisiológico e natural, que traz benefícios para a criança, a mãe, a família e a sociedade em geral (GIUGLIANI, 2000). É a principal estratégia na redução da mortalidade infantil, podendo evitar $13 \%$ das mortes por causas preveníveis em menores de cinco anos em todo o mundo. Segundo a Organização Mundial da Saúde (OMS) e o Unicef, cerca de seis milhões de crianças são salvas por ano graças ao Aleitamento Materno exclusivo (AME) (BRASIL, 2011).

Existem vários aspectos científicos que demonstram as vantagens do AM nas suas diferentes interfaces, tanto do ponto de vista nutricional, como do ponto de vista da proteção que o alimento confere, além dos aspectos emocionais, sociais e de prevenção de doenças (BRASIL, 2009a). A OMS recomenda a prática da amamentação exclusiva por seis meses e a manutenção do Aleitamento Materno acrescido de alimentos complementares até os dois anos de vida ou mais (BRASIL, 2002).

A prática da amamentação é determinada biologicamente, já que o corpo feminino está apto para amamentar, mas a decisão da mulher de fazê-lo, assim como a de mantê-lo, sofre influência das práticas sociais existentes no contexto em que vive e por isso deixou de ser praticada universalmente a partir do século XX. (CASTRO; ARAÚJO, 2006).

Dentro desta perspectiva, por meio de uma proposta de trabalho articulado em rede, o Ministério da Saúde lançou a Rede Amamenta Brasil, instituindo-a no âmbito do SUS por meio da Portaria No 2.799 de 18 de novembro de 2008, que preconiza uma estratégia de promoção, proteção e apoio à prática do Aleitamento Materno na atenção básica e tem como principal objetivo contribuir para redução da mortalidade materna e infantil através do aumento dos índices de Aleitamento Materno no País. (BRASIL, 2011).

Esta rede tem como estratégia a capacitação de profissionais que atuam em Unidades Básicas de Saúde (UBS) para que se tornem agentes de mudança no ensino e aprendizagem do AM, por meio de uma prática integrada baseada na educação permanente em saúde, a fim de discutir a prática e monitoramento dos índices de AM das UBS cadastradas, respeitando a visão de mundo dos profissionais e considerando as especificidades locais e regionais (BRASIL, 2009a).

A partir disto, o modelo da amamentação precisa ser modificado, pois esta prática deve ser compreendida não apenas nos aspectos biológicos, mas nas suas determinações sociais e culturais. 0 grande desafio dos profissionais de saúde é compreender que o ato de amamentar está inserido em um contexto sociocultural, pois atua junto à mulher, família e comunidade e para isso é necessário ter conhecimento dos aspectos históricos, sociais, culturais e biológicos da amamentação (BRASIL, 2009a).

O presente estudo objetivou descrever os indicadores de Aleitamento Materno em crianças de zero a seis meses de idade nas UBS que participam da Rede Amamenta Brasil no município de Aracaju/SE. Como também identificar os alimentos utilizados pelas crianças que não realizam o Aleitamento Materno exclusivo e verificar quais as ações desenvolvidas por essas unidades de saúde para o incentivo desta prática. 


\section{METODOLOGIA}

O município de Aracaju/SE tem sua rede de atenção primária composta por 136 Equipes de Saúde da Família (ESF) distribuídas em 43 UBS, mas a implantação da Rede Amamenta Brasil através da Oficina de Formação de Tutores em AM aconteceu em outubro de 2009 em cinco UBS. São elas: as UBSs Manoel de Souza Pereira, José Quintiliano, Carlos Hardman, Eunice Barbosa e João Bezerra.

A presente pesquisa foi desenvolvida na Secretaria Municipal de Saúde de Aracaju/SE, especificamente no Programa Saúde da Criança, Adolescente e Jovem (PSCAJ) que faz parte de uma das frentes da Coordenação de Vigilância em Saúde (Covis). E nas UBS João Bezerra no Bairro Mosqueiro; Eunice Barbosa na comunidade do Coqueiral, Bairro Porto Dantas; e Manoel de Souza Pereira, no Conjunto Sol Nascente, Bairro Jabutiana.

A UBS João Bezerra abrange uma população dependente do SUS 93\%, abastecimento de água proveniente de poço ou nascente com $89 \%$, apenas $2 \%$ das casas tem sistema de esgoto; a UBS Eunice Barbosa possui $99 \%$ da população dependente do SUS, baixa condição socioeconômica e falta de saneamento básico, onde apenas $21 \%$ das residências possuem sistema de esgoto; e a UBS Manoel de Souza Pereira possui $62 \%$ da população SUS dependente de saneamento básico adequado.

Trata-se de um estudo transversal descritivo, com base em dados contidos na Planilha Rede Amamenta Brasil que faz o acompanhamento de crianças de 1-15 meses que comparecem às Unidades Básicas de Saúde para vacinação de rotina (Anexo 1); e na Planilha mensal consolidada da Rede Amamenta Brasil (Anexo 2); como também um questionário estruturado (Apêndice 1).

Foram utilizados os dados presentes nas planilhas acima descritas nos períodos de janeiro a outubro do ano de 2012 nas UBS João Bezerra e Eunice Barbosa, e nos períodos de agosto a outubro do ano de 2012 na UBS Manoel de Souza Pereira, referentes às crianças de zero a seis meses de idade.

A coleta dos dados foi realizada em duas fases. $\mathrm{Na}$ primeira foram analisados os dados contidos na Planilha Rede Amamenta Brasil que faz o acompanhamento de crianças de 1-15 meses que comparecem às Unidades Básicas de Saúde para vacinação de rotina, através das seguintes variáveis: idade, leite materno, água, chá, suco de frutas, outros leites, fruta, comida salgada e outros alimentos. Esta pretendia verificar se as crianças estavam em Aleitamento Materno exclusivo, e, se não, identificar quais os alimentos utilizados.

O outro instrumento foi a análise dos dados contidos na Planilha mensal consolidada da Rede Amamenta Brasil que apresentam os seguintes indicadores: proporção de crianças em Aleitamento Materno, proporção de crianças recebendo água, proporção de crianças recebendo chá, proporção de crianças recebendo sucos ou bebidas à base de água, proporção de crianças recebendo outros leites, proporção de crianças recebendo alimentos sólidos ou semisólidos e proporção de crianças e Aleitamento Materno exclusivo.

A segunda fase foi realizada através de um questionário estruturado aplicado com cada equipe de saúde que faz parte das UBS. Este instrumento apresentava variáveis que dizem respeito à assistência e ao processo de trabalho em equipe, onde foi possível verificar as ações implantadas por essas unidades direcionadas à Rede Amamenta Brasil.

As variáveis relacionadas à assistência foram: realização de pré-natal; orientação sobre Aleitamento Materno; realização de consultas puerperal; e realização de visita domiciliar na primeira semana de vida. 
As variáveis relacionadas ao processo de trabatho em equipe são: existência de grupo de gestantes; abordagem do Aleitamento Materno no grupo de gestantes; fluxograma de atendimento da dupla mãe/ bebê em amamentação; garantia de consulta para mães com problemas em amamentação; encaminhamento para centro de referência; grupo de apoio à amamentação; realização de palestra/reunião mensal sobre Aleitamento Materno; ação sobre Aleitamento Materno no Conselho de Saúde ou em equipamentos sociais; monitoramento dos indicadores de Aleitamento Materno nas equipes; e a UBS realiza monitoramento e avaliação dos indicadores de Aleitamento Materno.
A análise de dados foi descritiva para as duas fases da pesquisa, através de frequências. Para confecção de gráficos e tabelas foi utilizado o programa Excel 2007- Microsoft Office.

O estudo obteve aprovação do Comitê de Ética e Pesquisa da Universidade Tiradentes, por meio do Protocolo $n^{\circ} 120912$, para coleta de dados na SMS de Aracaju/SE e nas UBS. De acordo com a resolução 196/96 do Conselho Nacional de Saúde, a pesquisa obedece todas as normas preconizadas para pesquisas com seres humanos.

\section{RESULTADOS}

Na primeira fase da pesquisa foram analisadas 742 as crianças de zero a seis meses de idade que estavam

cadastradas nas Planilhas da Rede Amamenta Brasil.

Tabela 1. Número de crianças cadastradas nas Planilhas das UBS no período de janeiro a outubro de 2012 no município de Aracaju/SE, Brasil.

\begin{tabular}{llll}
\hline Idade & UBS João Bezerra & UBS Eunice Barbosa & UBS Manoel de Souza \\
1 mês & 26 & 33 & 12 \\
2 meses & 57 & 74 & 41 \\
3 meses & 47 & 65 & 33 \\
4 meses & 36 & 52 & 31 \\
5 meses & 36 & 41 & 32 \\
6 meses & 37 & 68 & 21 \\
TOTAL & 239 & 333 & 170 \\
\hline
\end{tabular}

Fonte: Secretaria Municipal de Saúde de Aracaju/SE 
As UBS Eunice Barbosa e João Bezerra apresentam o maior número de crianças, pois os dados são referentes ao período de janeiro a outubro de 2012 e na
UBS Manoel de Souza Pereira diz respeito ao período de agosto a outubro do mesmo ano (Tabela 1).

Tabela 2. Indicadores das Planilhas da UBS João Bezerra no período de janeiro a outubro de 2012 no município de Aracaju/SE, Brasil.

\begin{tabular}{lllllllllll}
\hline Idade & AM $\%$ & AME $\%$ & Água $\%$ & Chá $\%$ & $\begin{array}{l}\text { Suco de } \\
\text { Frutas } \%\end{array}$ & $\begin{array}{l}\text { Outros } \\
\text { Leites } \%\end{array}$ & Frutas $\%$ & $\begin{array}{l}\text { Comida } \\
\text { Salgada } \\
\%\end{array}$ & $\begin{array}{l}\text { Outros } \\
\text { Alimentos } \\
\%\end{array}$ \\
\hline mês & $88 \%$ & $74 \%$ & $23 \%$ & $23 \%$ & $0 \%$ & $27 \%$ & $0 \%$ & $0 \%$ & $0 \%$ \\
2 meses & $95 \%$ & $52 \%$ & $44 \%$ & $25 \%$ & $0 \%$ & $40 \%$ & $0 \%$ & $0 \%$ & $0 \%$ \\
3 meses & $81 \%$ & $53 \%$ & $55 \%$ & $36 \%$ & $17 \%$ & $45 \%$ & $4 \%$ & $2 \%$ & $2 \%$ \\
4 meses & $83 \%$ & $43 \%$ & $64 \%$ & $42 \%$ & $39 \%$ & $58 \%$ & $6 \%$ & $8 \%$ & $6 \%$ \\
5 meses & $75 \%$ & $25 \%$ & $75 \%$ & $39 \%$ & $42 \%$ & $61 \%$ & $33 \%$ & $25 \%$ & $11 \%$ \\
6 meses & $78 \%$ & $14 \%$ & $86 \%$ & $68 \%$ & $70 \%$ & $81 \%$ & $65 \%$ & $57 \%$ & $22 \%$ \\
TOTAL & $84 \%$ & $46 \%$ & $58 \%$ & $38 \%$ & $26 \%$ & $52 \%$ & $17 \%$ & $14 \%$ & $6 \%$ \\
\hline
\end{tabular}

Fonte: Secretaria Municipal de Saúde de Aracaju/SE

Na UBS João Bezerra a maioria das crianças nos dois primeiros meses de vida está em AM 88\% e 95\%, mas destas $74 \%$ e $52 \%$ estão em AME. Existe a introdução precoce de alimentos líquidos como água $44 \%$, outros leites $40 \%$ e chá $25 \%$, principalmente do segundo mês de vida (Tabela 2).

Em relação ao terceiro mês de vida, constata-se que $81 \%$ estão em AM e destas $53 \%$ em AME. Já no quarto mês a maioria está em AM 83\%, mas houve uma redução na prevalência do AME 43\%. Nessas fai- xas etárias muitas crianças consomem água, outros leites e chá e há introdução de uma pequena quantidade de alimentos sólidos, como fruta, comida salgada e outros alimentos (Tabela 2).

No quinto e sexto mês de vida, apesar das crianças estarem em AM, a prevalência do AME diminuiu significativamente $25 \%$ e $14 \%$. Na dieta das crianças são oferecidos todos os alimentos apontados na pesquisa. Foi observado também que no sexto mês muitas crianças alimentam-se de comida salgada 57\% (Tabela 2). 
Tabela 3. Indicadores das Planilhas da UBS Eunice Barbosa no período de janeiro a outubro de 2012 no município de Aracaju/SE, Brasil.

\begin{tabular}{llllllllll}
\hline Idade & AM $\%$ & AME $\%$ & Água $\%$ & Chá $\%$ & $\begin{array}{l}\text { Suco de } \\
\text { Frutas } \%\end{array}$ & $\begin{array}{l}\text { Outros } \\
\text { Leites } \%\end{array}$ & Frutas $\%$ & $\begin{array}{l}\text { Comida } \\
\text { Salgada } \\
\%\end{array}$ & $\begin{array}{l}\text { Outros } \\
\text { Alimentos } \%\end{array}$ \\
\hline 1 mês & $94 \%$ & $65 \%$ & $30 \%$ & $33 \%$ & $0 \%$ & $27 \%$ & $0 \%$ & $0 \%$ & $0 \%$ \\
2 meses & $91 \%$ & $48 \%$ & $50 \%$ & $38 \%$ & $4 \%$ & $43 \%$ & $0 \%$ & $0 \%$ & $0 \%$ \\
3 meses & $89 \%$ & $45 \%$ & $58 \%$ & $43 \%$ & $11 \%$ & $51 \%$ & $3 \%$ & $2 \%$ & $2 \%$ \\
4 meses & $79 \%$ & $32 \%$ & $73 \%$ & $38 \%$ & $38 \%$ & $62 \%$ & $15 \%$ & $12 \%$ & $10 \%$ \\
5 meses & $78 \%$ & $13 \%$ & $90 \%$ & $66 \%$ & $66 \%$ & $68 \%$ & $46 \%$ & $39 \%$ & $37 \%$ \\
6 meses & $76 \%$ & $6 \%$ & $96 \%$ & $75 \%$ & $79 \%$ & $76 \%$ & $68 \%$ & $53 \%$ & $56 \%$ \\
TOTAL & $84 \%$ & $35 \%$ & $68 \%$ & $50 \%$ & $33 \%$ & $56 \%$ & $23 \%$ & $18 \%$ & $18 \%$ \\
\hline
\end{tabular}

Fonte: Secretaria Municipal de Saúde de Aracaju/SE

Na UBS Eunice Barbosa, apesar da maioria das crianças estarem em AM nos dois primeiros meses de vida, a prevalência do AME é baixa: $65 \%$ e $48 \%$. Foram introduzidos alimentos líquidos na dieta das crianças, tendo uma maior frequência no segundo mês, pois $50 \%$ das crianças bebem água e uma grande quantidade consome outros leites $43 \%$ e chá $38 \%$ (Tabela 3).

Nas faixas etárias do terceiro e quarto mês de vida, muitas estão em AM $89 \%$ e $79 \%$, mas poucas se alimentam de forma exclusiva: $45 \%$ e $32 \%$. São oferecidos em maior proporção os alimentos líquidos. No quarto mês, $73 \%$ consomem água e $62 \%$ outros leites. Existe uma pequena introdução dos alimentos sólidos (Tabela 3).

Em relação às crianças com cinco e seis meses de vida, o estudo mostrou que muitas estão em $A M$, mas a prevalência do AME é muito baixa: $13 \%$ e $6 \%$. No quinto mês muitas bebem água, $90 \%$, e mais da metade das crianças consome outros leites (68\%), chá (66\%) e suco de frutas (66\%). No sexto mês destaca-se o consumo de água (96\%), já o chá (75\%), suco de frutas (79\%) e outros leites (76\%) apresentam aproximadamente o mesmo quantitativo. Nesses meses percebe-se também um consumo muito grande de alimentos sólidos (Tabela 3). 
Tabela 4. Indicadores das Planilhas da UBS Manoel de Souza Pereira no período de agosto a outubro de 2012 no município de Aracaju/SE, Brasil.

\begin{tabular}{llllllllll}
\hline Idade & AM $\%$ & AME $\%$ & Água $\%$ & Chá $\%$ & $\begin{array}{l}\text { Suco de } \\
\text { Frutas } \%\end{array}$ & $\begin{array}{l}\text { Outros } \\
\text { Leites } \%\end{array}$ & Frutas $\%$ & $\begin{array}{l}\text { Comida } \\
\text { Salgada } \\
\%\end{array}$ & $\begin{array}{l}\text { Outros } \\
\text { Alimentos } \%\end{array}$ \\
\hline 1 mês & $92 \%$ & $100 \%$ & $8 \%$ & $8 \%$ & $8 \%$ & $8 \%$ & $8 \%$ & $8 \%$ & $0 \%$ \\
2 meses & $100 \%$ & $80 \%$ & $10 \%$ & $2 \%$ & $5 \%$ & $17 \%$ & $2 \%$ & $0 \%$ & $0 \%$ \\
3 meses & $94 \%$ & $77 \%$ & $18 \%$ & $6 \%$ & $15 \%$ & $21 \%$ & $9 \%$ & $3 \%$ & $0 \%$ \\
4 meses & $90 \%$ & $71 \%$ & $26 \%$ & $10 \%$ & $19 \%$ & $26 \%$ & $10 \%$ & $3 \%$ & $0 \%$ \\
5 meses & $91 \%$ & $52 \%$ & $22 \%$ & $22 \%$ & $47 \%$ & $31 \%$ & $31 \%$ & $22 \%$ & $0 \%$ \\
6 meses & $100 \%$ & $33 \%$ & $43 \%$ & $24 \%$ & $67 \%$ & $48 \%$ & $62 \%$ & $43 \%$ & $0 \%$ \\
TOTAL & $95 \%$ & $68 \%$ & $21 \%$ & $11 \%$ & $25 \%$ & $25 \%$ & $18 \%$ & $11 \%$ & $0 \%$ \\
\hline
\end{tabular}

Fonte: Secretaria Municipal de Saúde de Aracaju/SE

Na UBS Manoel de Souza, na faixa etária com um mês de vida, $92 \%$ estão em AM, destas $100 \%$ estão em AME. Percebe-se também que uma criança alimenta-se de água, chá, suco de frutas, outros leites, fruta e comida salgada (Tabela 4).

Em relação às crianças com dois meses de idade, observa-se que todas estão em AM, mas apenas $80 \%$ estão em AME. Uma pequena quantidade alimenta-se de outros leites (17\%), água (10\%), suco de frutas (5\%), chá (2\%) e fruta (2\%). Nesta faixa etária já existe a introdução do suco de frutas na alimentação (Tabela 4).
Nas crianças com três e quatro meses de vida, foi percebido que a maioria encontra-se em AM 94\% e $90 \%$ e muitos em AME $77 \%$ e $71 \%$. Uma pequena quantidade consome outros leites, água, e suco de frutas. Existe uma pequena introdução de alimentos sólidos como fruta e comida salgada (Tabela 4).

As crianças com cinco meses de vida constatam-se $91 \%$ está em AM, já as crianças com seis meses, $100 \%$. Apesar do alto índice de AM, houve uma redução considerável da prevalência do AME nesses meses: $52 \%$ e $33 \%$. Percebe-se um crescimento no consumo de suco de frutas, frutas e outros leites (Tabela 4). 
Tabela 5. Indicadores da Planilha Mensal Consolidada da Rede Amamenta Brasil no período de 2012 no município de Aracaju/SE, Brasil.

\begin{tabular}{|c|c|c|c|c|c|c|c|c|}
\hline \multirow[t]{2}{*}{ Alimentos } & \multicolumn{2}{|c|}{ João Bezerra } & \multicolumn{2}{|c|}{ Eunice Barbosa } & \multicolumn{2}{|c|}{ Manoel de Souza } & \multicolumn{2}{|c|}{ Total } \\
\hline & $\mathrm{N}^{\circ}$ & $\%$ & $\mathrm{~N}^{\circ}$ & $\%$ & $\mathrm{~N}^{\circ}$ & $\%$ & $\mathrm{~N}^{\circ}$ & $\%$ \\
\hline AM & 201 & $84 \%$ & 281 & $84 \%$ & 161 & $95 \%$ & 643 & $87 \%$ \\
\hline AME & 92 & $46 \%$ & 98 & $35 \%$ & 110 & $68 \%$ & 300 & $40 \%$ \\
\hline Água & 139 & $58 \%$ & 225 & $68 \%$ & 35 & $21 \%$ & 399 & $54 \%$ \\
\hline Chá & 91 & $38 \%$ & 172 & $52 \%$ & 19 & $11 \%$ & 282 & $38 \%$ \\
\hline Suco de Frutas & 63 & $26 \%$ & 111 & $33 \%$ & 43 & $25 \%$ & 217 & $29 \%$ \\
\hline Outros Leites & 124 & $52 \%$ & 186 & $56 \%$ & 43 & $25 \%$ & 353 & $48 \%$ \\
\hline Frutas & 40 & $17 \%$ & 75 & $23 \%$ & 31 & $18 \%$ & 146 & $20 \%$ \\
\hline C. Salgada & 34 & $14 \%$ & 59 & $18 \%$ & 19 & $11 \%$ & 112 & $15 \%$ \\
\hline O. Alimentos & 15 & $6 \%$ & 59 & $18 \%$ & 0 & $0 \%$ & 74 & $10 \%$ \\
\hline
\end{tabular}

Fonte: Secretaria Municipal de Saúde de Aracaju/SE

No que diz respeito aos dados contidos na PlaniIha Mensal Consolidada da Rede Amamenta Brasil, observamos algumas proporções dos indicadores.

Das 742 crianças, $87 \%$ estão em AM, no entanto $40 \%$ de forma exclusiva. Na UBS Manoel de Souza Pereira, $95 \%$ das crianças estão em AM e $68 \%$ em AME. As UBS João Bezerra e Eunice Barbosa tiveram a mesma porcentagem $84 \%$ em AM, mas a prevalência de $A M E$ foi superior na UBS João Bezerra com 46\% (Tabela 5).

No que diz respeito a outros alimentos, observa-se o maior consumo de água $54 \%$ e outros leites (48\%), seguido de chá (38\%) e suco de frutas (29\%). A introdução desses alimentos na dieta das crianças ocorre com uma maior frequência nas UBS Eunice Barbosa e na UBS João Bezerra, nestas mais da metade das crianças consomem água e outros leites. Cerca de $20 \%$ das crianças já se alimentam de comidas sólidas, como fruta, comida salgada e outros alimentos (Tabela 5).
$\mathrm{Na}$ segunda fase da pesquisa foram analisados oito questionários aplicados a cada equipe das UBS. Sendo dois na UBS João Bezerra; dois na UBS Eunice Barbosa; e quatro na UBS Manoel de Souza Pereira.

Em relação às variáveis relacionadas à assistência, constatamos que $100 \%$ das equipes realizam pré-natal na unidade, orientam as gestantes sobre o AM durante essas consultas e que realizam visita domiciliar para o trinômio mãe/filho/família na primeira semana pós-parto. Quanto à oferta de consulta puerperal, foi percebido que uma equipe da UBS João Bezerra não realiza.

No que tange as variáveis relacionadas ao processo de trabalho, verificamos que $100 \%$ das equipes realizam grupo de gestantes na UBS e que a temática do Aleitamento Materno sempre é abordada nesses grupos. A maioria das equipes $(87,5 \%)$ possui fluxograma de atendimento da dupla mãe/bebê em amamentação, sendo que apenas uma equipe da UBS João Bezerra não possui esse fluxograma. 
Foi percebido que $87,5 \%$ das equipes garantem a consulta no mesmo dia para mães e bebês com problemas na amamentação, sendo que uma equipe da UBS Eunice Barbosa não o faz e que apenas $50 \%$ das equipes realizam algum encaminhamento a um centro de referência quando mães e bebês apresentam problemas no processo da amamentação.

Em relação às ações educativas, constatamos que $100 \%$ das equipes realizam pelo menos uma palestra ou reunião sobre AM na UBS e que em $75 \%$ das unidades existe grupo de apoio à amamentação, com exceção da UBS Eunice Barbosa. Foi constatado que $62,5 \%$ das equipes desenvolvem alguma ação sobre
AM nos Conselho de Saúde ou em equipamentos sociais da comunidade.

No que diz respeito à realização do monitoramento dos indicadores de $A M$, a pesquisa mostrou que $87,5 \%$ das equipes acompanham esses indicadores, apenas uma equipe da UBS João Bezerra não monitora. Quanto à oferta de um espaço para reuniões entre as equipes da UBS a fim de monitorar e avaliar os indicadores de Aleitamento Materno, 62,5\% das equipes relatou ter acesso a esse espaço, sendo que na UBS Eunice Barbosa essas reuniões não existem e na UBS João Bezerra uma equipe também informou a não realização dessa reunião em sua unidade.

\section{DISCUSSÃO}

Considerando a recomendação da OMS que a prática da amamentação deve exclusiva por seis meses, o estudo mostrou que $87 \%$ estão em AM, todavia a prevalência do AME abrange apenas $40 \%$ destas crianças. Duas pesquisas nacionais de Prevalência de AM nas capitais brasileiras e no Distrito Federal realizadas no período de 1999 a 2008 evidenciaram que em 1999 a prevalência de AME em menores de quatro meses chegou a 35,6\%, enquanto em 2008 esta prevalência foi de $51,2 \%$ e em crianças menores de seis meses foram $41 \%$. Nesta a prevalência do AME foi heterogênea entre as regiões e capitais brasileiras, 0 Nordeste apresentou a pior situação 37,0\% e Aracaju/ SE 35,0\% (BRASIL, 2009b).

O estudo evidenciou que apesar das crianças estarem em AM 87\%, nas UBS João Bezerra e Eunice Barbosa poucas recebem de forma exclusiva, respectivamente $46 \%$ e $35 \%$. Já na UBS Manoel de Souza esse indicador é superior 68\%. Em 2002 no estudo realizado em Londrina (PR), a prevalência do AME foi $21 \%$ (VANNUCHI et al, 2005). Em 2004, no município de Cuiabá, Mato Grosso 34,5\% (FRANÇA et al, 2007).

Já no município de Joinville (SC), em 2005 a prevalência de AM foi de $84,2 \%$ e o índice de AME 43,6\% (FRANCO et al, 2008). E em 2007, no município do Rio de Janeiro, foram 58,1\% (PEREIRA et al, 2010).

A pesquisa mostrou que nos dois primeiros meses de vida os indicadores de AME na UBS João Bezerra foram respectivamente $74 \%$ e $52 \%$, na UBS Eunice Barbosa $65 \%$ e $48 \%$ simultaneamente e na UBS Manoel de Souza Pereira $100 \%$ e $80 \%$. Um estudo realizado em 2005, nos Núcleos de Saúde da Família da cidade de Ribeirão Preto, São Paulo (SP), os lactentes estavam em AME com $76,8 \%$ e $52,9 \%$ nos 30 e 60 dias (KISHI et. al., 2009).

Em todas as unidades foi observada a introdução precoce de água, chá e outros leites na alimentação das crianças no primeiro mês de vida, mas é partir do segundo mês que essa introdução acontece com uma maior frequência. Uma pesquisa nacional realizada no Brasil em 2008 também constatou que no primeiro mês de vida existia a introdução precoce desses alimentos (BRASIL, 2009b). 
O estudo apontou que as crianças com faixa etária entre três e quatro meses estão em $A M$, sendo que na UBS João Bezerra os indicadores de AME foram respectivamente $53 \%$ e $43 \%$, na UBS Eunice Barbosa $45 \%$ e $32 \%$ e na UBS Manoel de Souza Pereira $77 \%$ e $71 \%$ ao mesmo tempo. Em um estudo realizado em 2004, no município de Cuiabá (MT) a prevalência de AME foi de $41 \%$ em menores de quatro meses (FRANÇA et al, 2007). Em 2005, no município de Ribeirão Preto (SP) foi 31,4\% de AME no terceiro mês de vida e 12,1\% no quarto mês (KISHI et. al., 2009). Já em 2005 no município de Joinville (SC), a prevalência do AME nas crianças menores de quatro meses de idade foi de $53,9 \%$ (FRANCO et al, 2008).

O estudo também mostrou que nessas faixas etárias houve um aumento no consumo dos líquidos, e uma introdução pequena de alimentos sólidos como fruta, comida salgada e outros alimentos na alimentação das crianças em todas as UBS. A pesquisa nacional realizada em 2008 também evidenciou a iniciação da fruta e da comida salgada na dieta das crianças entre três e seis meses de idade (BRASIL, 2009b).

Quanto ao quinto e sexto mês de idade, nas UBS João Bezerra e Eunice Barbosa $77 \%$ das crianças estão em AM, mas de forma exclusiva o quantitativo é muito baixo $25 \%$ e $08 \%$ respectivamente, já na UBS Manoel de Souza Pereira, 94\% estão em AM e 44\% em AME. Em um estudo realizado em 2003 no município de Conchas (SP), a prevalência do AME em menores de seis meses foi heterogênea, variando de 7,4 a 41,2\% (PARADA et. al., 2005). Um estudo nacional realizado 2012, o qual observou que houve aumento na prevalência do AM no Brasil, mas ainda assim são poucas as regiões com mais de $50 \%$ das crianças com menos de seis meses recebendo leite materno ( $\mathrm{PO}$ ZZEBOM et. al., 2012).
Com a análise dos questionários, a pesquisa mostrou que na UBS Manoel de Souza Pereira o acompanhamento ao trinômio mãe/filho/família e o incentivo ao Aleitamento Materno acontecem de forma efetiva, através das ações assistenciais e educativas. Os indicadores de Aleitamento Materno são elevados e monitorados pelas equipes. Esses fatores podem estar relacionados ao fato desta UBS estar localizada em um bairro considerado de classe média, com $62 \%$ da população SUS dependente e com saneamento básico adequado.

Na UBS João Bezerra, as equipes realizam ações direcionadas à assistência, mas não encaminham a um centro de referência mães e bebês com problemas no processo da amamentação e não desenvolvem ação sobre AM nos Conselho de Saúde ou em equipamentos sociais da comunidade. A não efetivação dessas pode estar correlacionada à dificuldade de acesso a UBS, pois está localizada em um povoado com uma área geográfica extensa e quem mora mais longe não se desloca para esse tipo de ação. Nesta unidade a prevalência do AME foi baixa (46\%), embora $93 \%$ dessa população dependam do SUS.

Na UBS Eunice Barbosa apesar da população depender do SUS $99 \%$, os indicadores de AME foram muito baixos (35\%). Esses indicadores podem ser justificados pela baixa condição socioeconômica e falta de saneamento básico do bairro, como também a não realização de algumas ações na unidade, entre elas, grupo de apoio à amamentação, monitoramento dos indicadores de AM, desenvolvimento de ação sobre AM nos Conselho de Saúde ou em equipamentos sociais da comunidade e encaminhamento a um centro de referência quando mães e bebês apresentam problemas no processo da amamentação. 


\section{CONCLUSÃO}

O estudo demonstrou que $87 \%$ das crianças estão em AM, todavia a prevalência do AME é baixa (40\%). Isso se deve à introdução precoce de outros tipos de alimentos e líquidos na alimentação, o que pode ser desvantajoso, já que estes são menos nutritivos que o leite materno, podendo ocasionar aos lactentes dificuldades na absorção de nutrientes necessários para o seu desenvolvimento, desnutrição, ocorrência de doenças e diminuição do vínculo mãe e filho.

Os dados dos questionários evidenciaram que as UBS realizam o incentivo e a orientação por meio do pré-natal, puericultura, grupos de gestante e ações educativas, como também desempenham o acompanhamento assistencial ao trinômio mãe/filho/família. $E$ que apenas $50 \%$ das equipes realizam algum encaminhamento a um centro de referência quando mães e bebês apresentam problemas no processo da amamentação.

Esse fato demonstra que alguns profissionais precisam ser capacitados quanto ao manejo e à prevenção dos principais problemas que podem ocorrer durante o período da amamentação, a fim de que algumas dificuldades sejam resolvidas na UBS. Como também é necessário sensibilizá-los para o aconselhamento, já que o ato de amamentar está inserido em um contexto sociocultural, devendo o profissional ter conhecimento dos aspectos históricos, sociais, culturais e biológicos da amamentação, possibilitando assim não só adesão à prática, mas a manutenção por períodos mais longos.

O monitoramento dos indicadores de AM é um prérequisito para participar da Rede Amamenta Brasil, por esse motivo a pesquisa mostra que estes indicadores são monitorados pelos profissionais de saúde, mas apenas $62,5 \%$ das UBS oferecem espaço para reuniões com as equipes a fim de monitorar e avaliar esses indicadores. Faz-se necessário o acompanha- mento do tutor da Rede Amamenta Brasil de forma efetiva para trabalhar os indicadores e contribuir com o planejamento das ações a serem desenvolvidas nas UBS em benefício da amamentação.

Vale destacar que de acordo com o Ministério da Saúde haverá a integração da Rede Amamenta Brasil e a Estratégia Nacional para Alimentação Complementar Saudável (Enpacs), na qual assume a denominação "Estratégia Amamenta e Alimenta Brasil", lançada em 28 de abril de 2012. Esta estratégia visa à qualificação do processo de trabalho dos profissionais da atenção básica, o fortalecimento das ações de Aleitamento Materno e da alimentação saudável para crianças menores de dois anos, no âmbito do Sistema Único de Saúde (SUS).

Portanto, o estudo evidencia que apesar das UBS que participam da Rede Amamenta Brasil promoverem ações para o $A M$, esta prática não acontece de forma exclusiva e o desmame precoce se configura cada vez mais como um problema de saúde pública, para tanto, cabe ao poder público, ações mínimas efetivas, incentivando a promoção, proteção e apoio ao Aleitamento Materno, a integração entre os serviços de saúde e divulgação dos indicadores de AM.

Há necessidade de continuar realizando estudos sobre a temática, devido à escassez de dados referentes à promoção do $A M$ na atenção primária. Pois a vigilância a partir de dados e pesquisa contribui para revelar a importância do AME e fornecer informações para tomadas de decisões na esfera do SUS.

Espera-se que este estudo sensibilize os gestores e profissionais de saúde para o desenvolvimento de ações conjuntas a fim de avaliar os serviços ofertados e planejar ações de incentivo à prática do Aleitamento Materno. 


\section{REFERÊNCIAS}

BRASIL. Ministério da Saúde. Dez passos para uma alimentação saudável. Guia alimentar para crianças menores de 2 anos. Um guia para o profissional da saúde na atenção básica. Brasília: Ministério da Saúde, 2002.

BRASIL. Ministério da Saúde. Portaria GM/MS n. 2.799. Institui a Rede Amamenta Brasil. Diário Oficial da União, 19 nov. 2008.

BRASIL. Ministério da Saúde. Secretaria de Atenção à Saúde, Departamento de Ações Programáticas Estratégicas. Rede Amamenta Brasil: caderno do tutor. Brasília: Ministério da Saúde, 2009a.

BRASIL. Ministério da Saúde. Secretaria de Atenção à Saúde, Departamento de Ações Programáticas e Estratégicas. II Pesquisa de Prevalência de Aleitamento Materno nas Capitais Brasileiras e Distrito Federal. Brasília: Ministério da Saúde, 2009b.

BRASIL. Ministério da Saúde. Secretaria de Atenção à Saúde, Área Técnica de Saúde da Criança e Aleitamento Materno. Rede Amamenta Brasil: os primeiros passos (2007-2010). Brasília: Ministério da Saúde, 2011.

CASTRO, LMCP de, ARAÚJO, LDS de. Aspectos socioculturais da amamentação. In: Aleitamento Materno: manual prático. 2.ed. Londrina:PML, 2006. Cap. II, p. 37-40.

FRANÇA, G.V.A; BRUNKEN, G.S; SILVA, S.M; ESCUDER,M.M; VENÂNCIO, S.I. Determinantes da amamentação no primeiro ano de vida em Cuiabá, Mato Grosso. Rev Saúde Pública 2007;41(5):711-18.

FRANCO, S.C; NASCIMENTO, M.B.R; REIS, M.A.M; ISSLER,H; GRISI,S.J.F.E. Aleitamento Materno exclusivo em lactentes atendidos na rede pública do município de Joinville, Santa Catarina, Brasil. Rev. Bras. Saúde Materno Infantil, Recife, 8 (3): 291-297, jul. / set., 2008.

GIUGLIANI, E.R.J. 0 Aleitamento Materno na prática clínica. Jornal de Pediatria, Rio de Janeiro, vol. 76, supl.3, 2000.

KISHI, R.G.B; CACCIA-BAVA, M.G.G; MARTINEZ, E.Z. Prevalência do Aleitamento Materno exclusivo e fatores associados entre as crianças menores de 6 meses cadastradas em unidades de saúde da família. Rev. APS, v. 12, n. 1, p. 54-61, jan./mar. 2009

PARADA, C.M.G.L; CARVALHAES, M.A.B.L; WINCKLER, C.C; WINCKLER, L.A; WINCKLER V.C. Situação do Aleitamento Materno em população assistida pelo programa de saúde da família-PSF. Rev Latino-am Enfermagem 2005 maio-junho; 13(3):407-14.

PEREIRA, R.S.V; OLIVEIRA, M.I.C; ANDRADE, C.L.T; BRITO, A.S. Fatores associados ao Aleitamento Materno exclusivo: o papel do cuidado na atenção básica. Cad. Saúde Pública, vol.26, n.12, Rio de Janeiro, Dec. 2010. 
POZZEBON, N.M; FREIRE, A.B; REAL, A.A; BRAZ, M.M; PIVETTA, H.M.F; PRESTES, M.Lúcia. Prevalência de Aleitamento Materno exclusivo no Brasil. Trabalho vinculado ao programa de Auxílio à pesquisa Recém-doutor (ARD/CCS/UFSM), 2012.

VANNUCHI, M.T.O; THOMSON, Z; ESCUDER, M.M.L; TACLA, M.T. G. M; VEZOZZO,K.M. K; CASTRO, L.M. C. P; OLIVEIRA, M.M. B; VENANCIO, S.I. Perfil do Aleitamento Materno em menores de um ano no Município de Londrina, Paraná. Rev. Bras. Saúde Materno Infantil, vol.5 no.2, Recife Apr./Junho 2005. 
Recebido em: 24 de Maio de 2013 Avaliado em: 5 de Agosto de 2013 Aceito em: 8 de Agosto de 2013
1 Assistente Social, residente em Saúde Coletiva pela Universidade Tiradentes, Aracaju, Sergipe, Brasil. E-mail: lilianessiqueira@hotmail.com

2 Assistente Social, tutora da Residência Multiprofissional Integrada em Saúde Coletiva da Universidade Tiradentes, mestre em Saúde e Ambiente na mesma universidade, Aracaju, Sergipe, Brasil. E-mail: anaceliagoes@ hotmail.com

3 Enfermeira, coordenadora do Sistema de Informação da Secretaria Municipal de Saúde de Aracaju, mestre em Saúde e Ambiente pela Universidade Tiradentes, Aracaju, Sergipe, Brasil. E-mail: renata.silva@aracaju. se.gov 